\title{
Viscoelastic Properties of Comb-Shaped Polystyrenes Having Parent Polymers of Different Molecular Weights
}

\author{
Teruo Fujimoto, Hirokazu Kajiura, Masuhiko Hirose, and Mitsuru Nagasawa \\ Department of Synthetic Chemistry, Nagoya University, \\ Chikusa-ku, Nagoya, Japan.
}

(Received June 7, 1971)

\begin{abstract}
Comb-shaped polystyrenes having parent polymers of different molecular weights were prepared by an anionic polymerization method. A series of those samples have branches of a molecular weight lower than the critical value for entanglement. The samples were fractionated and characterized by osmotic pressure and lightscattering methods. The steady-flow viscosity, the steady-state compliance and other rheological parameters were calculated from the data of stress relaxation measurements of those samples. The effects of entanglement between parent polymers and of that between branches on the rheological properties are discussed from the molecular weight dependence of steady-flow viscosity, based on the theories of Bueche and Graessley.

KEY WORDS Comb-Shaped Polymer / Branched Polymer / Stress

Relaxation / Steady-Flow Viscosity / Steady-State Compliance /

Entanglement / Graessley / Bueche /
\end{abstract}

In a previous paper, ${ }^{1}$ it was experimentally shown that the steady-flow viscosity of combshaped poly styrenes, relative to that of the corresponding linear polystyrenes with the same molecular weight, is primarily determined by the radius of gyration of the sample, though, strictly speaking, the difference in the state of entanglement between comb-shaped and linear polymers must be taken into account. In the paper, the entanglement between two parent polymers was assumed to be negligible because of low fractions of parent polymer in the samples. However, if the molecular weight of parent polymer is in creased or if the molecular weight of branches is decreased, so that the fraction of parent polymer in the samples may increase, entanglement between parent polymers may become predominant in comparison with that between branches.

The purpose of this paper is to clarify the effects of the entanglement between parent polymers on the viscoelastic properties of combshaped polymers by measuring stress relaxation of comb-shaped polymers of various molecular weights.

\section{EXPERIMENTAL}

\section{Samples}

Preparation, fractionation, and characterization of comb-shaped polystyrene samples as well as purification of monomer and solvents, preparation of $n$-butyllithium and cumyl potassium were carried out in the same way as described in a previous paper. ${ }^{1}$ Number-average molecular weights of the parent polystyrenes used in this work are 5.6, 37, and $78 \times 10^{4}$, while it was $9.5 \times 10^{4}$ in the previous work. ${ }^{1}$ Because of the experimental difficulty, the molecular weights of branches could not be kept at a constant value but they are between 6 and $15 \times 10^{4}$. In addition, one sample has branches of $1.8 \times 10^{4}$ molecular weight, which is lower than the critical molecular weight for entanglement $\left(M_{\mathrm{c}}\right) ;(3-4) \times 10^{4}$ for linear polystyrenes ${ }^{2-4}$ and $(3.5-4) \times 10^{4}$ proposed for branches of combshaped polystyrenes. ${ }^{1}$ The number of branches in a molecule was calculated from the weightaverage molecular weight of the sample and the number-average molecular weight of a branch. Those molecular weights were determined from light scattering and osmotic pressure measurements, respectively, as described in a previous paper $^{1}$. A molecular weight of a branch $1.8 \times 10^{4}$ 
was calculated from the limiting viscosity number in toluene at $25^{\circ} \mathrm{C}$ using the equation ${ }^{5}$

$$
[\eta]=34.5 \times 10^{-5} M_{w}^{0,62}
$$

The weight-average molecular weights $\left(M_{w}\right)$ and the numbers of branches $\left(N_{\mathrm{b}}\right)$ of the present samples are listed in Table I, together with the

Table I. The samples

\begin{tabular}{lcccc}
\hline Series & Sample no. & $M_{w} \times 10^{-6}$ & $N_{\mathrm{b}}$ & $g$ \\
\hline Linear & $\mathrm{L}$ & $0.36_{8}$ & 0 & 1 \\
\hline K6-Series & $\mathrm{K} 6-4$ & $2.5_{5}$ & 18.8 & $0.15_{1}$ \\
$M_{n, \mathrm{p}}=5.7_{3} \times 10^{4}$ & -6 & $1.7_{5}$ & 12.7 & $0.24_{1}$ \\
& -10 & $1.5_{1}$ & 10.9 & $0.28_{5}$ \\
$M_{n, \mathrm{~b}}=1.3_{2} \times 10^{5}$ & -11 & $1.0_{4}$ & 7.5 & $0.37_{9}$ \\
\hline K40-Series & $\mathrm{K} 40-8$ & $9.0_{9}$ & 138 & $0.060_{1}$ \\
$M_{n, \mathrm{p}}=3.6_{8} \times 10^{5}$ & -11 & $5.9_{2}$ & 87.8 & $0.091_{8}$ \\
$M_{n, \mathrm{~b}}=6.3_{2} \times 10^{4}$ & -16 & $3.5_{6}$ & 50.5 & $0.14_{9}$ \\
\hline $\mathrm{K} 402-$ Series & $\mathrm{K} 402-1$ & $2.7_{8}$ & 38.0 & $0.18_{8}$ \\
$M_{n, \mathrm{p}}=3.6_{8} \times 10^{5}$ & -3 & $1.0_{9}$ & $40 . \mathrm{I}$ & $0.36_{5}$ \\
$M_{v, \mathrm{~b}}=1.8 \times 10^{4}$ & -4 & $0.85_{5}$ & 27.1 & $0.44_{9}$ \\
\hline K80-Series & -5 & $0.62_{2}$ & 14.1 & $0.61_{1}$ \\
$M_{w, \mathrm{p}}=7.8_{3} \times 10^{5}$ & $\mathrm{~K} 80-1$ & 14.8 & 95.4 & $0.087_{0}$ \\
$M_{n, \mathrm{~b}}=1.4_{7} \times 10^{5}$ & -4 & $6.5_{7}$ & 39.4 & $0.18_{7}$ \\
& -6 & $5.5_{9}$ & 32.7 & $0.22_{1}$ \\
& & $4.7_{5}$ & 26.7 & $0.25_{4}$ \\
\hline
\end{tabular}

ratio of unperturbed mean radius of gyration of the branched polymer to that of the corresponding linear polymer having the same molecular weight, $g_{\mathrm{s}}$, which is calculated from the equation of Orofino and Berry. ${ }^{6,7}$

A monodisperse polystyrene of $M_{w}=78 \times 10^{4}$, which was used as the parent polymer for series K80, was purchased from Pressure Chemical Co.

The reliability of the molecular structure for the samples in series K-6 was confirmed by comparing the observed distribution of branch density in the unfractionated sample with the expected distribution calculated from the mechamism of coupling reaction as in a previous paper, ${ }^{1}$ but that for other samples was confirmed simply by observing that the molecular weights of those fractionated samples were lower than the maximum molecular weight expected.

\section{Stress Relaxation Measurements}

The method of making sample films and the measurements of stress relaxation are the same as in a previous paper. ${ }^{8}$ The samples in series K402 give very brittle films.

Determination of Glass Transition Temperature

The glass transition temperature was determined by a conventional method, raising the temperature of an oil bath with a heating rate of $1^{\circ} \mathrm{C} / 4 \mathrm{~min}$.

\section{RESULTS}

The change in relaxation modulus of sample K402-3 with logarithmic time at different temperatures is shown as an example in Figure 1.

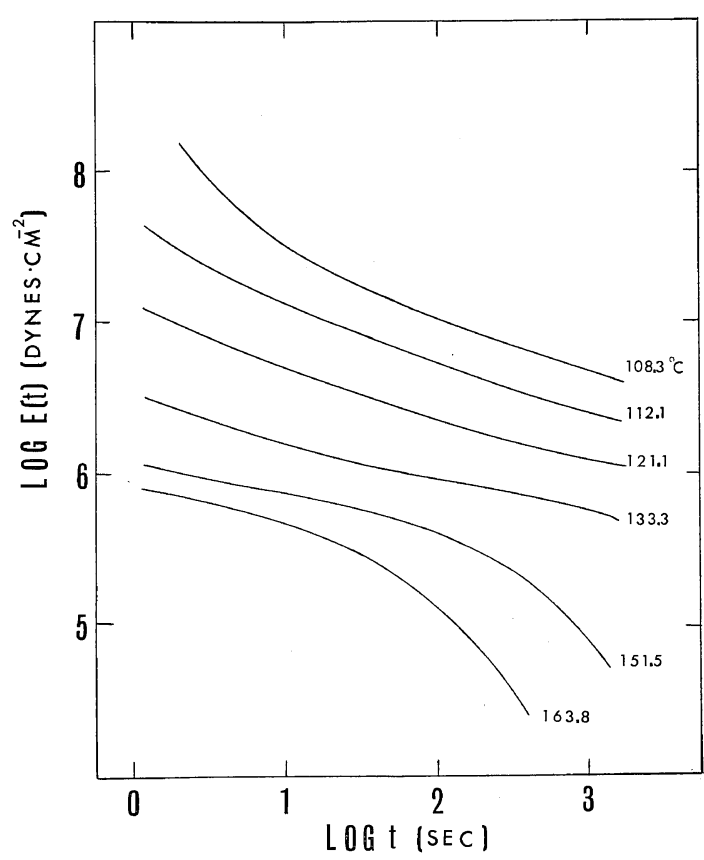

Figure 1. Experimental relaxation modulus plotted logarithmically against time at various temperatures (sample K 402-3).

The correction for the change in density with temperature is taken into account in the figure. The densities of the samples at different temperatures are assumed to be equal to those of linear polymers. ${ }^{9}$ All data at various temperatures are shifted to that at a reference temperature of $140^{\circ} \mathrm{C}$ according to the time-temperatue 
superposition principle. $^{10}$ The shift factors $a_{\mathrm{T}}$ plotted against temperature for all samples including sample series K402 fit the curve of linear polystyrenes, independent of the density of branches and the molecular weight of a branch, as shown in Figure 2. The curve

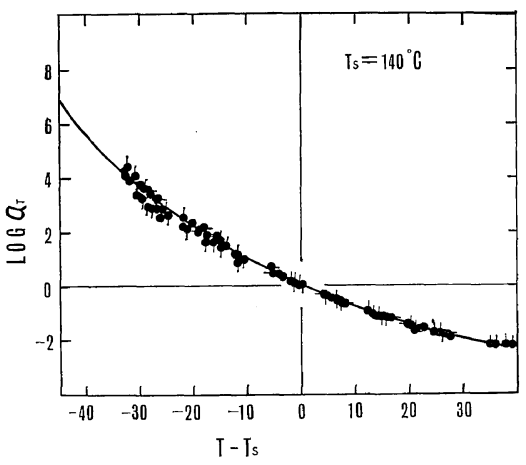

Figure 2. The shift factor $a_{\mathrm{T}}$ for all samples: K6; - K 40; - , K 402; ', K80. The solid line denotes the original WLF equation.

shown represents the original WLF equation having two universal constants $C_{1}=8.86$ and $C_{2}=101.6$.

The master curves obtained by the superposition for some typical samples are shown in Figure 3. As reported in the previous paper, ${ }^{1}$ the comb-shaped polystyrenes have a relaxation modulus lower than the linear polystyrenes in

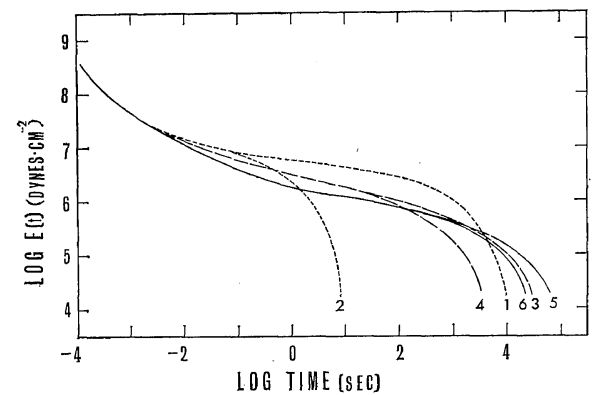

Figure 3. Master curves for some typical samples. The dash line 1 denotes the experimental data for the linear polystyrene $L$. The dashed line 2 denotes the data for a linear polystyrene of $M_{w}=$ $6.3_{2} \times 10^{4}$ estimated from the data in ref 2 . The broken and solid lines 3, 4, 5, and 6 show the data for $\mathrm{K} 40-8, \mathrm{~K} 40-17$, $\mathrm{K} 402-1$, and $\mathrm{K} 402-3$, respectively. the rubbery plateau region. Moreover, the relaxation moduli of the samples in series K 402 are rather lower than not only those of linear polystylenes but also those of the other combshaped polystyrenes in the rubbery plateau region. In the terminal zone, the master curves of sample series $\mathrm{K} 402$ extend to a longer time region than those of the other samples, despite the fact that the molecular weights of those samples are much lower than those of the other comb-shaped samples.

The relaxation spectrum $\log H(\tau)$ can be calculated from the master curves in Figure 3. using the second approximation method of Ferry and Williams, ${ }^{11}$ as shown in Figure 4 . The

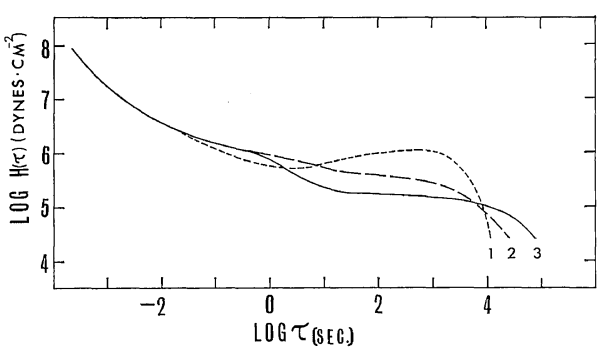

Figure 4. Relaxation spectra for some typical samples. The dashed line 1 , the broken line 2, and solid line 3 show the data for linear polystyrene L, comb-shaped polystyrene K40-11 and K402-1, respectively.

relaxation spectra of comb-shaped polystyrenes in the present work as well as in the previous work $^{1}$ are qualitatively similar to those for polydisperse linear polystyrenes, ${ }^{10,12}$ except the one for sample series K402. A shoulder is found around $\log \tau \simeq 0.5$ on the spectra for K402.

The steady-flow viscosity $\eta$ and the steadystate compliance $J_{\mathrm{e}}$ can be calculated from the stress relaxation master curves in Figure 3 and equations ${ }^{10}$

$$
\begin{aligned}
& \eta=(1 / 3) \int_{-\infty}^{\infty} t E(t) \mathrm{d} \ln t \\
& J_{\mathrm{e}}=\left(1 / 3 \eta^{2}\right) \int_{-\infty}^{\infty} t^{2} E(t) \mathrm{d} \ln t
\end{aligned}
$$

The values of $\eta$ obtained are shown in Figure 5 and Table II. They are much lower than 


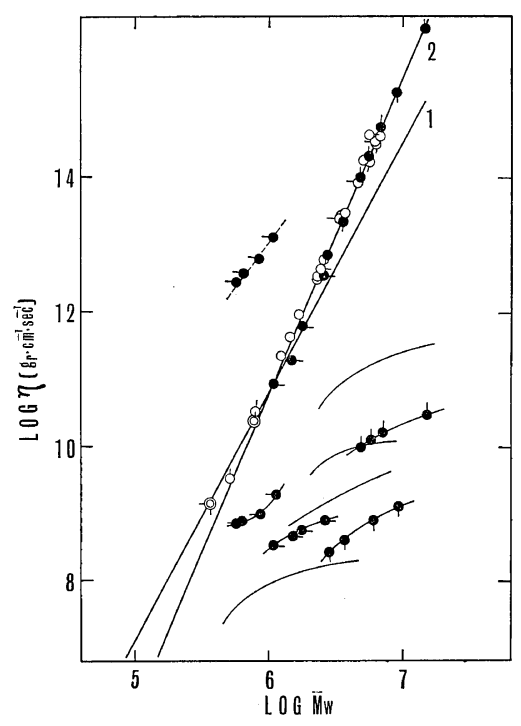

Figure 5. Molecular weight dependence of the steady-flow viscosity. Symbols are the same as in Figure 2. The solid curves denote data in a previous paper. ${ }^{1}$ The solid line 1 shows the molecular weight dependence of $\eta_{l}$ of linear polystyrenes. ${ }^{13,14}$ The symbol -(9) shows the value of $\eta_{l}$ for linear polystyrene $\mathbf{L}$ observed in this work, whereas () shows the estimated value of $\eta_{l}$ a linear polystyrene with $M_{w}=7.8_{3} \times 10^{5}$. The solid straight line 2 denotes the molecular weight dependence of $\eta_{l} i$ for imaginary linear polystyrenes. The filled circles are observed in the present work, while the open circles are the data reported in ref 1 . The four points on a dotted line show the values of $\eta_{l}^{i}$ for series $\mathrm{K} 402$.

the viscosities of linear polymers having the same molecular weights. The viscosity does not so markedly increase with the increase in the number of branches if the molecular weight of branches is kept constant. These results are in accord with the results reported in a previous paper. ${ }^{1}$ Here, however, it is to be noted that the viscosities of the samples in series $\mathrm{K} 40$ and $\mathrm{K} 80$ are lower than those of these of their parent linear polystyrenes. It is also noteworthy that $\eta$ of sample series $\mathrm{K} 402$ increases with increasing branch number. The molecular weight dependence of $\eta$ for linear polystyrenes in Figure 5 was reproduced from the data of Tobolsky and Akovali. ${ }^{13,14}$ The value of $\eta$ for a monodisperse polystyrene of $M_{w}=36 .{ }_{8} \times 10^{4}$,
Table II. Rheological data

\begin{tabular}{rcccc}
\hline Sample no. & $\begin{array}{c}\tau_{\mathrm{m}} \times 10^{-4}, \\
\text { sec }\end{array}$ & $\begin{array}{c}E_{\mathrm{m}} \times 10^{-5}, \\
\mathrm{dyn} / \mathrm{cm}^{2}\end{array}$ & $\begin{array}{c}\eta \times 10^{-9}, \\
\mathrm{~g} / \mathrm{cm} \mathrm{sec}\end{array}$ & $\begin{array}{c}J_{\mathrm{e}} \times 10^{6}, \\
\mathrm{~cm}^{2} / \mathrm{dyn}\end{array}$ \\
\hline K $6-4$ & $0.20_{9}$ & 14 & $1.2_{2}$ & 1.3 \\
-6 & $0.63_{0}$ & 2.1 & $0.50_{2}$ & 4.9 \\
-10 & $0.45_{6}$ & 1.9 & $0.37_{2}$ & 4.2 \\
-11 & $0.40_{7}$ & 2.0 & $0.34_{9}$ & 5.5 \\
\hline $\mathrm{K} 40-8$ & $0.34_{7}$ & 1.7 & $0.25_{1}$ & 5.7 \\
-11 & $1.3_{2}$ & 2.0 & $1.2_{5}$ & 7.8 \\
-16 & $0.83_{2}$ & 1.8 & $0.89_{1}$ & 7.2 \\
-17 & $0.46_{8}$ & 2.0 & $0.42_{7}$ & 6.7 \\
\hline $\mathrm{K} 402-1$ & $0.28_{2}$ & 1.7 & $0.27_{4}$ & 6.8 \\
-3 & $2.8_{6}$ & 1.6 & $1.9_{8}$ & 10 \\
-4 & $1.2_{6}$ & 1.7 & $0.98_{0}$ & 10 \\
-5 & $0.97_{2}$ & 1.9 & $0.86_{6}$ & 8.2 \\
\hline K 80 - 1 & $0.79_{4}$ & 1.7 & $0.73_{7}$ & 7.5 \\
-4 & 40.2 & 1.9 & 31.5 & 7.1 \\
-6 & 20.1 & 2.5 & 15.5 & 7.2 \\
-8 & 16.1 & 2.0 & 13.1 & 7.0 \\
\hline & 11.7 & 2.6 & 10.2 & 6.8 \\
\hline
\end{tabular}

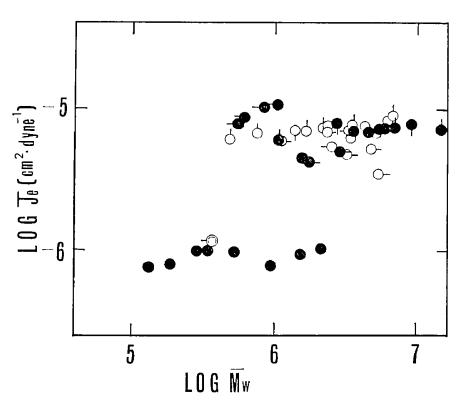

Figure 6. Molecular weight dependence of the steady-state compliance. The symbols are the same as in Figures 2 and 5 . The filled circles for linear polystyrenes are reproduced from ref 13 .

used as a parent polymer, was determined in this work and is shown by a double circle in Figure 5.

The steady-state compliance $J_{\mathrm{e}}$ of the samples obtained is shown in Figure 6 and in Table II. As in a previous paper, ${ }^{1} J_{\mathrm{e}}$ of comb-shaped polystyrenes is much higher than that of linear polystyrenes. Moreover, $J_{e}$ of $\mathrm{K} 402$ is also higher than that of linear polymers and even those of other comb-shaped polystyrenes.

The maximum relaxation time $\tau_{\mathrm{m}}$ and the 
modulus associated with the maximum relaxation time $E_{\mathrm{m}}$ are obtained by the same method as in the previous work $^{1}$ and are shown in Table II and in Figures 7 and 8, respectively.

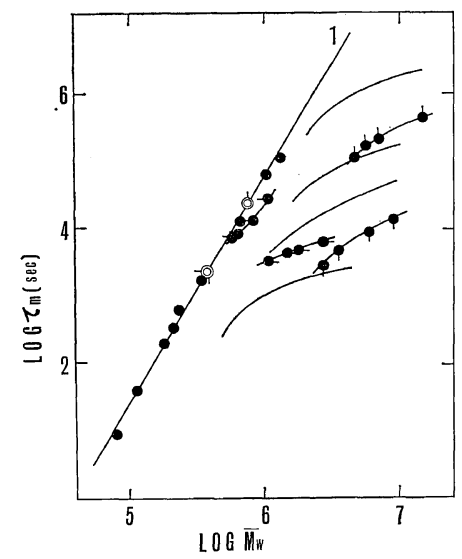

Figure 7. Molecular weight dependence of the maximum relaxation time. The symbols are the same as in Figures 2 and 5.

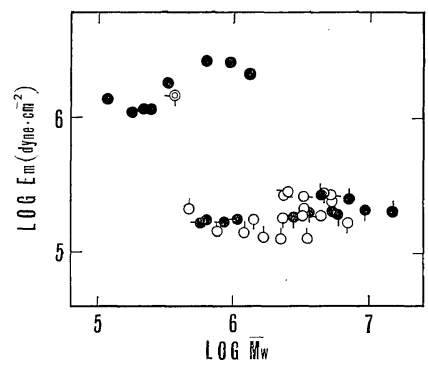

Figure 8. Molecular weight dependence of the relaxation modulus associated with the maximum relaxation time. The symbols are the same as in Figures 2, 5, and 6 .

The specific volume-temperature curves for K402-3 and K40-16 are shown in Figure 10. The glass transition temperatures are $95.0^{\circ} \mathrm{C}$ and $95.6^{\circ} \mathrm{C}$ for $\mathrm{K} 402-3$ and $\mathrm{K} 40-16$, respective1y. No significant difference is observed between the volume recovery curves of K402-3, K40-16 and linear polystyrenes, determined by the method of Kovacs. ${ }^{15,16}$

\section{DISCUSSION}

According to Bueche ${ }^{17}$ and also to Graessley, ${ }^{18}$ if the theory of Graessley for linear polymers

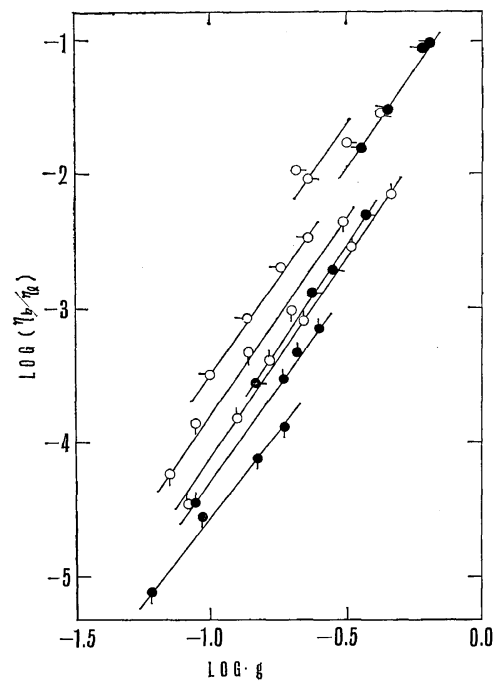

Figure 9. Plots of $\log \left(\eta_{\mathrm{b}} / \eta_{1}\right)$ vs. $\log g_{\mathrm{s}}$ for combshaped polystyrenes. The symbols are the same as in Figures 2 and 5. The average slope is. 2.9 (see ref 19).

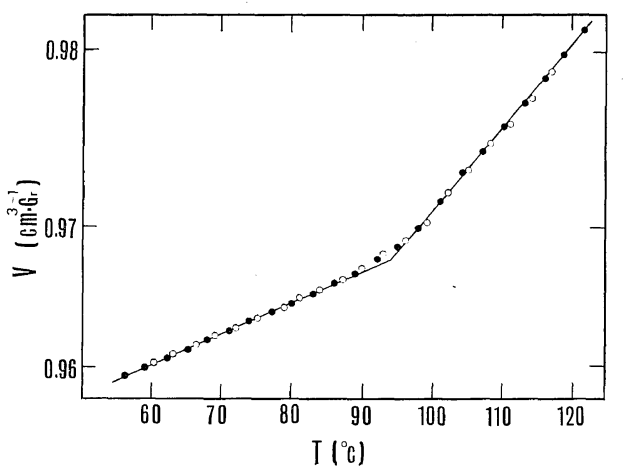

Figure 10. The specific volume-temperature curves for K 402-3 and K40-16. The filled and open circles show the specific volumes of $\mathrm{K} 402-3$, and $\mathrm{K} 40-16$, respectively. The heating rate is $1^{\circ} \mathrm{C} / 4$ $\min$.

may be extended to comb-shaped ${ }^{1}$ polymers, the ratio of the steady-flow viscosity of a combshaped polymer to that of the corresponding linear polymer having the same molecular weight, $\eta_{\mathrm{b}} / \eta_{1}$, is given by the 3.5 th power of the ratio of their radii of gyration $g_{\mathrm{s}}$. That is, ${ }^{17}$

$$
\eta_{\mathrm{b}} / \eta_{1}=g_{\mathrm{s}}{ }^{7 / 2}
$$

The equation of Graessley for the steady-flow 
viscosity of linear polymers $\left(\eta_{1}\right)$ and that of comb-shaped polymers $\left(\eta_{\mathrm{b}}\right)^{1}$ may be written as

$$
\begin{aligned}
& \eta_{l}=K\left\langle S^{2}\right\rangle_{l}\left[1+0.131\left(M / M_{\mathrm{e}}\right)^{5 / 2}\right] \\
& \eta_{\mathrm{b}}=K\left\langle S^{2}\right\rangle_{\mathrm{b}}\left[1+0.305\left(M_{\mathrm{b}} / M_{\mathrm{e}}\right)^{5 / 2}\right]
\end{aligned}
$$

where the suffixes $l$ and $b$ specify the linear and branched polymers, respectively, $\left\langle S^{2}\right\rangle$ the radius of gyration, $M$ the molecular weight of the sample, $M_{\mathrm{b}}$ the molecular weight of a branch $M_{\mathrm{e}}$ the molecular weight between two adjacent entanglement points (assumed to be $2 \times 10^{4}$ ) and $K$ is a constant.

Equation 4 is expected to be applicable irrespective of "the number of branches and the molecular weight of a branch. Neither the data in the previous paper $^{1}$ nor the data in the present work, however, give a single straight line in the $\log \left(\eta_{\mathrm{b}} / \eta_{1}\right)$ vs. $\log g_{\mathrm{s}}$ plots. The plot appears linear only if the molecular weight of branches is kept unchanged, as shown in Figure 9. The average slope is about 2.9 instead of 3.5. ${ }^{*}$ As discussed previously, ${ }^{1}$ the discrepancy may be attributed to the fact that the state of entanglement in branched polymers may be different from that in linear polymers. Thus, if there were a linear polymer with the same state of entanglements as the branched polymers, the ratio of the viscosity of the branched polymer to that of such an imaginary linear polymer having the same molecular weight $\eta_{\mathrm{b}} / \eta_{1}{ }^{\mathrm{i}}$ could be explained by the theories of Graessley and Bueche. We further estimate the viscosity of the imaginary linear polymer $\eta_{1}{ }^{i}$ by multiplying the culculated ratio $\eta_{1} / \eta_{\mathrm{b}}$ by the observed value of $\eta_{\mathrm{b}}$ based on the above expectation. $\eta_{1}{ }^{i}$ thus estimated is found to fall along a straight line in the $\log \eta_{1}{ }^{i}$ vs. $\log M$ plot with a slope higher than that for linear polymers. Except for the points of series K402, all data in the present work are consistent with the data in a previous paper $^{1}$ irrespective of the molecular weight of parent polymer as shown Figure 5. The points for K402 do not fall along the

* There was an error in the drawing of Figure 11 in ref 1 . The explanations for $\log g_{\mathrm{s}}$ and $\log \left(\eta_{\mathrm{b}} / \eta_{1}\right)$ in the figure should be exchanged and the values on the abscissa should be multiplied by a factor of 0.1. Then the slope of the solid lines in the figure is 2.8. Figure 9 in the present paper is the corrected figure. line but show higher values than the points for $\mathrm{K} 40$, despite the fact that both $\mathrm{K} 402$ and $\mathrm{K} 40$ have the same parent polymer. The reason might be such that the length of branches of $\mathrm{K} 402$ is so short that the effects of entanglement between the parent polymers cannot be neglected, whereas it is negligible for $\mathrm{K} 40$ as well as for other samples.

Here it is important that the samples in series K40 and K80 have lower viscosities than their parent polymers. This fact also shows that entanglement between parent polymers in those samples in negligible and their viscosities are governed by the entanglement on branches. (There may be entanglement between parent polymer and branches, but the entanglement gives almost the same effect on $\eta$ as the entanglement between branches. They need not usually be distinguished). In the samples of $\mathrm{K} 402$, on the other hand, there may be both entanglement between parent polymers and between parent polymer and branches though there would be no effective entanglement between branches, since the fraction of parent polymer in those samples is fairly large (23$30 \%$ ). The above conclusion may also be supported by the fact that the relaxation spectra for the samples in series K402 have a shoulder around $\log \tau \simeq 0.5$, as seen in Figure 4. That is, the entanglement between parent polymers would contribute to the terminal zone, while entanglement of branches would cause the shoulder if the molecular weight of the parent is higher than that of the branches. It is observed in Figure 4 that the relaxation spectra for the samples in series K402 extend towards a longer relaxation time region than those for series $\mathrm{K} 40$, despite the fact that the molecular weights of the samples as well as the molecular weight of their branches in series $\mathrm{K} 402$ are much lower than in K40. A similar shoulder in the relaxation spectrum was reported for mixtures of two monodisperse polymers by Masuda, Kitagawa, Inoue, and Onogi. ${ }^{19}$ Here, it is to be noted that the terminal zone of K40 is located almost in the same region as that of their parent. This is, however, just a coincidence since such a feature cannot be found for other samples in present and previous works. ${ }^{1}$ It is posible that the relaxation spectrum of a 
comb-shaped polymer extends towards a longer relaxation time region than that for either its parent polymer or its branch polymer, but cannot extend to a longer relaxation time region than that for a linear polymer having the same molecular weight as the comb-shaped polymer (neglecting the difference in the states of entanglement for both polymers ${ }^{1}$ ).

Thus, it can be said for branched polymers that the contribution of entanglement between parent polymers to the bulk viscoelasticity should be discussed in relation to that between branches. As the molecular weight of branches increases, the entanglement between branches would increase but the entanglement between parent polymers would decrease, so that the effects of entanglement between branches may become predominant for the viscosity for such samples. If the molecular weight of branches is lower than that of the parent polymer, therefore, the steady-flow viscosity would decrease with increasing number of branches. The increase in the number of branches, however, would also result in the increase in viscosity because of the reason described in the theories of Bueche and Graessley. Because of these counteractive effects, the dependence of viscosity on branch number may go through a minimum provided the molecular weight of branches is much lower than that of the parent polymer. Such a tendency may be observed in the experimental data for series $\mathrm{K} 402$ and also for $\mathrm{K} 80$ and $\mathrm{K} 40$.

In a previous paper, ${ }^{1}$ it was suggested that if the molecular weight of branches is lower than the critical value for entanglement $\left(4 \times 10^{4}\right)$, the viscosity of the sample would always decrease with an increasing number of branches. That is, the branches would act as a diluent for entanglement between parent polymers. This would also be true even when the molecular weight of branches is higher than the critical value if the molecular weight of branches is much lower than the molecular weight of parent polymer. Even if the molecular weight of branches is lower than the critical value, however, the brances may not always act as a simple diluent since entanglement between parent polymer and branches cannot disappear and the viscosity may be governed by the entanglement between the whole polymer molecules. This may be supported by the fact that $\eta_{\mathrm{b}}$ of the samples in series K402 increases with increasing branch density in Figure 5. In the steady-flow viscosity of linear polymers there is a discontinuous transition from an entangled state to the isolated state of polymers at a critical molecular weight, ${ }^{2-4}$ but in the viscosity of combshaped polystyrenes no such discontinuous transition would be found if the molecular weight of either the parent polymer or the branches is higher than a critical value.

Although the samples in series $\mathrm{K} 402$ have many short branches of $M=1.8 \times 10^{4}$, the free valumes of the samples would be almost equal to that of a linear polymer with $M=(4-6) \times$ $10^{4}$, since the branch has only one free end. In practice, no difference is found between the samples of $\mathrm{K} 402$ and others for their master curves in the transition region (Figure 3). Moreover, no significant differences in both the specific volumes and glass transition temperatures are found between $\mathrm{K} 402-3$ and $\mathrm{K} 40-16$ (Figure 10).

Finally the conclusions obtained in the previous work ${ }^{1}$ are all consistent with the present experimental results, including that $J_{e}$ for the comb-shaped polystyrenes is much higher than that for linear polymers and that the values of $M_{\mathrm{e}}$ calculated from $J_{\mathrm{e}}$ and $E_{\mathrm{e}}$ by applying the theory of rubber elasticity do not agree with each other.

Acknowlegement. We wish to thank Mr. Shun-iti Kobayashi for his assistance in measuring the specific volume-time relationship of the samples. This work was supported in part by a grant from Toyota Physical and Chemical Research Institute.

\section{REFERENCES}

1. T. Fujimoto, H. Narukawa, and M. Nagasawa, Macromolecules, 3, 57 (1970).

2. S. Onogi, T. Masuda, and K. Kitagawa; ibid., 3, 109 (1970).

3. G. C. Berry and T. G Fox, Adv. Polym. Sci., 5, 261 (1968).

4. R. A. Stratton, J. Colloid Interfac. Sci., 22, 517 (1966).

5. R. N. Mukherjea and P. Remmp, J. Chim. 
Phys., 56, 94 (1959).

6. T. A. Orofino, Polymer, 2, 305 (1961).

7. G. C. Berry and T. A. Orofino, J. Chem. Phys. 40, 1614 (1964).

8. T. Fujimoto, N. Ozaki, and M. Nagasawa, $J$. Polym. Sci., Part A, 6, 129 (1968).

9. R. S. Spencer and G. D. Gilmore, J. Appl. Chem., 20, 502 (1949).

10. J. D. Ferry, "Viscoelastic Properties of Polymers," John Wiley and Sons, Inc., New York, N.Y., 1961.

11. J. D. Ferry and M. L. Williams, J. Colloid Sci., 7, 347 (1952).

12. A. V. Tobolsky and K. Murakami, J. Polym.
Sci., 40, 443 (1959).

13. A. V. Tobolsky, J. J. Aklonis, and G. Akovali, J. Chem. Phys., 42, 723 (1965).

14. G. Akovali, J. Polym. Sci., Part A-2, 5, 875 (1967).

15. A. J. Kovacs, J. Polym. Sci., 30, 131 (1958).

16. H. Endo, T. Fujimoto, and M. Nagasawa, $J$. Polym. Sci., Part A-2, 7, 1669 (1969).

17. F. Bueche, J. Chem. Phys., 40, 484 (1964).

18. W. W. Graessley, ibid., 43, 2696 (1965); 47, 1942 (1967).

19. T. Masuda, K. Kitagawa, T. Inoue, and S. Onogi, Macromolecules, 3, 116 (1970). 